\title{
Treatment delay and radiological errors in patients with bone metastases
}

Correspondence

K. Ichinohe

Department of Radiotherapy

Fukuroi Municipal Hospital

2515-1 Kunou

Fukuroi, Shizuoka

Japan

Fax: $+81-538-43-2511$

$\ldots \ldots \ldots \ldots \ldots \ldots$

Received September 20, 2002

Accepted August 19, 2003

. . . . . . . . . . . . . . .

\author{
Departments of ${ }^{1}$ Radiotherapy and ${ }^{2}$ Radiology, Fukuroi Municipal Hospital, \\ Fukuroi, Shizuoka, Japan \\ ${ }^{3}$ Department of Radiology, Seirei Hospital, Hamamatsu, Shizuoka, Japan
}

\begin{abstract}
During routine investigations, we are surprised to find that therapy for bone metastases is sometimes delayed for a considerable period of time. To determine the extent of this delay and its causes, we reviewed the medical records of symptomatic patients seen at our hospital who had been recently diagnosed as having bone metastases for the last four years. The treatment delay was defined as the interval between presentation with symptoms and definitive treatment for bone metastases. The diagnostic delay was defined as the interval between presentation with symptoms and diagnosis of bone metastases. The results of diagnostic radiological examinations were also reviewed for errors. The study population included 76 males and 34 females with a median age of 66 years. Most bone metastases were diagnosed radiologically. Over $75 \%$ of patients were treated with radiotherapy. The treatment delay ranged from 2 to 307 days, with a mean of 53.3 days. In 490 radiological studies reviewed, we identified $166(33.9 \%)$ errors concerning $62(56.4 \%)$ patients. The diagnostic delay was significantly longer for patients with radiological errors than for patients without radiological errors $(\mathrm{P}<0.001)$, and much of it was due to radiological errors. In conclusion, the treatment delay in patients with symptomatic bone metastases was much longer than expected, and much of it was caused by radiological errors. Considerable efforts should therefore be made to more carefully examine the radiological studies in order to ensure prompt treatment of bone metastases.
\end{abstract}

\section{Introduction}

The prognosis of patients with bone metastases is generally poor (1), but several treatment methods have been proposed to improve their quality of life (1-3). Patients complaining of symptoms attributable to bone metastases should therefore be treated as promptly as possible. During routine studies, we were sur-
Key words

- Bone metastases

- Delay

- Radiology

- Error

- Radiotherapy prised to find that therapy for bone metastases is sometimes delayed for a considerable period of time. Many investigators have studied the causes of delays in treating primary malignancies (4-10), but the reasons for delays in treating bone metastases have yet to be considered. Thus, the aim of this study was to determine the delay in treatment of bone metastases and the reasons for this delay. 


\section{Material and Methods}

Between May 1997 and August 2001, 214 symptomatic patients with bone metastases were registered in the radiation department of our hospital. Of these patients, 110 who first presented at our hospital and who had been recently diagnosed as having bone metastases were included in the study. The main presenting symptoms, the date of presentation to a physician, the date of diagnosis, the diagnostic methods used, the date of treatment, medications, and the treatment methods were obtained from the medical records of each patient. The quality of life of the patients was measured with the Karnofsky index of performance status (PS) at first presentation, at the time of diagnosis of bone metastases, during treatment, and at the time of maximum effect after treatment.

The diagnostic delay was calculated as the interval between presentation to a physician with symptoms and diagnosis of bone metastases. The treatment delay was calculated as the interval between presentation to a physician with symptoms and initiation of definitive treatment for bone metastases. For the patients treated with radiotherapy, the interval between referral for irradiation and initiation of radiotherapy was calculated.

The results of radiological work-ups for diagnosis were also reviewed, focusing on the images where primary cancer, bone metastases or associated findings had been misdiagnosed. The decision of misdiagnosis was based on the consensus of two radiologists (N. Tooyama and M. Takahashi) who were not aware of the patients' histories or symptoms. According to the criteria of Kelvin et al. (11), radiological errors were classified as purely perceptive (lesions visible in retrospect), purely technical (lesions not seen in retrospect), interpretative (lesions observed at the time of study but malignancy not included in the differential diagnosis), and technical and perceptive (technical inad- equacy contributing to perceptive error). The reasons for these delays were also evaluated.

The Mann-Whitney test and the test for proportions were used to evaluate statistical significance.

\section{Results}

The study population included 76 males and 34 females, with a median age of 66 years (range 36-84 years) at presentation. The main presenting symptoms were back pain $(\mathrm{N}=52)$, pain in the extremities $(\mathrm{N}=$ $20)$, thoracic pain $(\mathrm{N}=14)$, pelvic pain $(\mathrm{N}=$ $10)$, muscle weakness $(\mathrm{N}=6)$, numbness in the extremities $(\mathrm{N}=4)$, and others $(\mathrm{N}=4)$. The primary sites were the lung $(\mathrm{N}=40)$, hepatobiliary system $(\mathrm{N}=18)$, gastrointestinal tract $(\mathrm{N}=18)$, prostate $(\mathrm{N}=14)$, breast $(\mathrm{N}=6)$, urinary tract $(\mathrm{N}=4)$, others $(\mathrm{N}=6)$, and unknown $(\mathrm{N}=4)$.

Diagnosis of bone metastases was confirmed by a bone biopsy $(\mathrm{N}=6)$ or radiological examination $(\mathrm{N}=104)$, including magnetic resonance imaging $(\mathrm{N}=44)$. Thirtyeight patients had a single bone metastasis, while 72 had multiple bone metastases. Fortyfour patients had a single or multiple spinal metastasis. In 40 (36.4\%) patients, symptoms of bone metastases were the first sign of malignancy. The remaining 70 patients were diagnosed as having bone metastases following treatment of their primary tumors. Treatments included radiotherapy $(\mathrm{N}=94)$, orthopedic surgery $(\mathrm{N}=6)$, systemic therapy with hormonal or cytotoxic agents $(\mathrm{N}=6)$, and orchiectomy $(\mathrm{N}=4)$.

Eighty-eight patients were supported with analgesic agents (35 patients with nonsteroidal anti-inflammatory drugs, 25 with opioid drugs, 16 with a combination of the two types of drugs, and 12 with others). At presentation, analgesic agents had been prescribed to 74 patients, with $60(81.1 \%)$ of them having presumptive diagnoses of degenerative or other benign diseases.

The diagnostic and treatment delays for 
each patient are shown in Table 1. The diagnostic delay was responsible for most of the treatment delay. Six patients were treated with radiotherapy after surgery. Overall, 100 patients were treated with radiotherapy and the interval between referral to the radiation department and initiation of radiotherapy ranged from 0 to 19 days, with a mean of 1.7 days. Six patients were irradiated urgently because of progressive neurological deficits.

For the diagnosis of bone metastases, 490 radiological work-ups were performed with a mean of 4.5 radiological studies per patient. These studies consisted of plain radiography $(\mathrm{N}=214)$, computed tomography $(\mathrm{CT}, \mathrm{N}=94)$, bone scintigraphy $(\mathrm{N}=82)$, magnetic resonance imaging $(\mathrm{N}=78)$, and other imaging studies $(\mathrm{N}=22)$. Hospital radiologists examined $168(34.3 \%)$ of the reports concerning these patients. These were CT $(\mathrm{N}=46)$, bone scintigraphy $(\mathrm{N}=60)$, magnetic resonance imaging $(\mathrm{N}=58)$, and other imaging studies $(\mathrm{N}=4)$.

On reviewing all of these radiological studies, $166(33.9 \%)$ errors were identified (plain radiography $=100 ; \mathrm{CT}=22 ;$ magnetic resonance imaging $=20$; bone scintigraphy $=16$; other $=8)$ concerning $62(56.4 \%)$ patients. In 24 of these errors, a variety of primary tumors (lung cancer, colon cancer, rectal cancer, renal cell carcinoma, pancreatic cancer), and associated findings (paraaortic lymphadenopathy, malignant pleural effusion) were overlooked in 18 patients. Of 166 errors, $18(10.8 \%)$ were the responsibility of hospital radiologists.

Of all errors, 108 (65.0\%) were purely perceptive, $44(26.5 \%)$ were interpretative, eight $(4.8 \%)$ were perceptive and technical, and six $(3.6 \%)$ were purely technical. With respect to 70 patients with a history of malignant disease, 70 radiological errors were identified in $34(48.6 \%)$ and $24(34.3 \%)$ were purely perceptive.

In one patient, radial bone metastasis was overlooked in a bone scintigraphy but a bone radiography performed on the same day revealed the metastasis. Another patient with severe back pain was first diagnosed as having a benign compression fracture of the thoracic spine on a chest X-ray, but a routine chest X-ray on admission revealed a lung tumor and he was correctly diagnosed as having a thoracic metastasis by $\mathrm{CT}$ of the chest on the same day. In a third patient, a lumbar metastasis was misinterpreted as degeneration by bone radiography, but an abdominal CT scan performed on the same day due to concomitant abdominal discomfort revealed the lumbar metastasis and colon cancer. The radiological errors concerning these three patients did not seem to have been responsible for the delayed diagnosis. All patients with radiological errors except these three were subsequently required to undergo observation with or without analgesics until the correct diagnosis had been made. Table 1 shows that the diagnostic delay in patients with radiological errors was much longer than that in those without, and that much of the diagnostic delay was due to the radiological errors.

A decrease in PS of $10 \%$ or more oc-

Table 1. Treatment delay and its causes $(N=110)$

\begin{tabular}{lcc}
\hline & Range (days) & Mean (days) [\%] \\
\hline Diagnostic delay (a) & $0-307$ & $39.0[73.2]$ \\
with radiological error $(\mathrm{N}=62)$ & $2-307$ & $54.2^{*+}$ \\
without radiological error $(\mathrm{N}=48)$ & $0-69$ & $19.4^{*}$ \\
Interval between diagnosis and treatment $(\mathrm{b})$ & $0-77$ & $14.3[26.8]$ \\
& $2-307$ & $53.3[100]$
\end{tabular}

*The mean diagnostic delay with radiological error was about 2.8 times longer than the delay without radiological error $(P<0.001$, Mann-Whitney test $)$.

+Once a patient was misdiagnosed by radiology, he had to wait an average of 46.2 more days until correct radiological examinations were performed (two were doublechecked and correctly diagnosed by a radiologist 1 and 5 days later). These intervals comprised $85.2 \%$ of the mean diagnostic delay with radiological errors, and $66.8 \%$ of the mean diagnostic delay of all patients. During these intervals, two patients refused further diagnostic work-ups for 7 and 44 days. Further radiological work-ups for two patients were postponed for 49 and 118 days because of previous treatment for complications (malignant pleural effusion and renal failure). The delays in these four patients made up $6.5 \%$ of the mean diagnostic delay. 
curred significantly more frequently in patients with radiological errors than in patients without radiological error $(33 / 62=$ $53.2 \%$ vs $15 / 48=31.3 \%, \mathrm{P}=0.02$ ) between presentation and diagnosis. Especially in 18 patients with local pain, radiological errors caused progression of symptoms; 12 of these patients were totally paralytic, and six immobile because of pathologic fracture of the pelvis or the femur at correct diagnosis.

After treatment, a $10 \%$ or higher increase of PS in patients with and without radiological errors was obtained in $70 \%$ and in $77 \%$ of the patients, respectively $(\mathrm{P}=0.47)$.

\section{Discussion}

The treatment delay in symptomatic patients with bone metastases was found to range from 2 to 307 days with a mean of 53.3 days. Nearly three quarters $(73.2 \%)$ of the treatment delay was due to diagnostic delay (Table 1). Although we could not find any previous reports of treatment delay for bone metastases in the literature, the delay in our patients was much longer than we would have expected.

Radiological errors occurred frequently in our study. About one third (33.9\%) of the radiological studies for diagnostic work-ups were misdiagnosed and these errors were identified in $54.6 \%$ of our patients. As also reported in previous studies $(5,10,11)$, these radiological errors caused long delays in diagnosis (Table 1). Furthermore, these errors often reduced the quality of life of our patients. In the present study, the symptoms of 18 patients who had first presented with only local pain had progressed to spinal paralysis or pathological fracture at diagnosis owing to radiological errors. It was disappointing that as many as $42.2 \%$ of all radiological errors were identified in $48.6 \%$ of patients with a history of malignant disease, and that over one third $(34.3 \%)$ of these errors were purely perceptive.

Since radiology is an important tool for evaluating patients with bone metastases $(12,13)$, it is clear that radiological studies should be conducted more carefully. We regret that in Japan radiologists do not interpret all radiological studies. In the present study, radiologists interpreted only $34.3 \%$ of radiological work-ups. To decrease errors, we believe radiologists should be involved more in radiological studies.

The effect of delay due to radiological errors on the PS of the patients was evident in this study. Between presentation and diagnosis, PS for the patients with radiological errors had decreased more frequently than for the patients without radiological error $(\mathrm{P}=0.02)$.

For the patients without radiological errors, improvement of PS after treatment was slightly more frequent than for the patients with radiological errors, but the difference was not significant. This may be due to the fact that sites of primary cancer, distributions of metastases, and treatment options also influence treatment outcomes. This is outside the scope of the present study.

The effect of using biochemical markers of bone metabolism for a prompt diagnosis of bone metastases was not evaluated in this study. In our opinion, these investigations are not as important as radiological studies for the prompt diagnosis of bone metastases because laboratory studies are often nonspecific for bone metastases (14) and radiological studies are mandatory for identifying metastatic bone lesions (12).

The interval between diagnosis and treatment showed that there was only a small treatment delay (Table 1). Although no comparable study is available, we are satisfied that the mean interval between referral to the radiation department of our hospital and initiation of radiotherapy was only 1.7 days. The prompt initiation of radiotherapy may be attributed to the fact that relatively small numbers of patients were irradiated each day with a 10-MV linear accelerator, with a staff of two radiation technologists and one radia- 
tion oncologist during the study period.

It should be noted that this study has several limitations: i) Pharmacological methods were not considered to be a definitive treatment for bone metastases. This is due to the fact that, although a patient with bone metastases may be treated with drugs only $(1-3,15)$, the initiation of such therapy might be based on an incorrect diagnosis of nonmalignant disease. At presentation, analgesics had been prescribed to over three fourths $(81.1 \%)$ of our patients without a correct diagnosis of bone metastases. If pharmacological therapies were regarded as definitive treatments of bone metastases, more bias would be introduced in estimating the effect of radiological errors on treatment delay. ii) It should be noted that the error rate in the present study did not necessarily reflect the accuracy of radiological examinations in our hospital. This is because false-positive findings of radiological examinations were not evaluated in the study design, and because not all radiological examinations performed during the study period were evaluated. iii) Other factors which may delay diagnosis include the delay in follow-up consultations, the time spent to present radiological examinations to physicians, and the waiting times for radiological examinations. Although the exact effect of these factors was not known, the policy of our hospital did not change during the 5-year period of this study; CT had to be performed and interpreted within a few days, plain radiography within one day, magnetic resonance imaging and bone scintigraphy within one week. In emergency situations, $\mathrm{CT}$ or magnetic resonance imaging had to be performed and interpreted within one day. Therefore, the effect of these factors is likely to have been small. iv) The small number of patients in this study might not represent all symptomatic patients with bone metastases. The patients were limited to those who presented themselves and were diagnosed at our hospital because the full medical records of these patients could easily be obtained.

Despite these limitations, the definitive treatment delays could be calculated from the medical records and the causes of these delays could be defined. Although the causes of these delays may differ among hospitals, it is important for physicians to know the exact treatment delay at their own hospital and to clarify its causes. The key to successful management of cancer patients is early diagnosis and prompt treatment $(7-10,16)$. Therefore, considerable efforts should be made to diagnose and treat these patients as quickly as possible. Continual monitoring of the treatment delay will be a useful indicator of the success of our efforts.

\section{References}

1. Lote K, Walløe A \& Bjersand A (1986). Bone metastasis: prognosis, diagnosis and treatment. Acta Oncologica, 25: 227-232.

2. Coleman RE (2000). Management of bone metastases. Oncologist, 5: $463-470$.

3. Schachar NS (2001). An update on the nonoperative treatment of patients with metastatic bone disease. Clinical Orthopaedics and Related Research, 382: 75-81.

4. Stebbing JF \& Nash AG (1995). Avoidable delay in the management of carcinoma of the right colon. Annals of the Royal College of Surgeons of England, 77: 21-23.

5. Zilling TL, Walther BS \& Ahren BO (1990). Delay in diagnosis of gastric cancer: a prospective study evaluating doctors' and patients' delay and its influence on five year survival. Anticancer Research, 10: 411-416.
6. MacArthur C, Pendleton L \& Smith A (1985). Treatment delay in patients with bladder tumours. Journal of Epidemiology and Community Health, 39: 63-66.

7. Silva PPA, Pereira JR, Ikari FK \& Minamoto H (1992). Lung cancer and the delay in diagnosis: analysis of 300 cases. Revista da Associação Médica Brasileira, 38: 145-149.

8. Robinson E, Mohilever J, Zidan J \& Sapir D (1986). Colorectal cancer: Incidence, delay in diagnosis and stage of disease. European Journal of Cancer and Clinical Oncology, 22: 157-161.

9. Rossi S, Cinini C, Pietro CD, Lombardi CP, Crucitti A, Bellantone R \& Crucitti $F$ (1990). Diagnostic delay in breast cancer: correlation with disease stage and prognosis. Tumori, 76: 559-562.

10. Joensuu H, Asola R, Holli K, Kumpulainen E, Nikkanen V \& Parvinen LM (1994). Delayed diagnosis and large size of breast cancer after a 
false negative mammogram. European Journal of Cancer, 30: 12991302.

11. Kelvin FM, Gardiner R, Vas W \& Stevenson GW (1981). Colorectal carcinoma missed on double contrast barium enema study: a problem in perception. American Journal of Roentgenology, 37: 307313.

12. Gold RI, Seeger LL, Bassett LW, Bassett LW \& Steckel RJ (1990). An integrated approach to the evaluation of metastatic bone disease. Orthopedics, 28: 471-483.

13. Rybak LD \& Rosenthal DI (2001). Radiological imaging for diagnosis of bone metastases. Quarterly Journal of Nuclear Medicine, 45: 53-
64

14. Meijer WG, Van Der Veer E \& Willemse PHB (1998). Biochemical parameters of bone metabolism in bone metastases of solid tumors (Review). Oncology Reports, 5: 5-21.

15. Ripamonti C \& Fulfaro F (2001). Pathogenesis and pharmacological treatment of bone pain in skeletal metastases. Quarterly Journal of Nuclear Medicine, 45: 65-77.

16. Helweg-Larsen S (1996). Clinical outcome in metastatic spinal cord compression: a prospective study of 153 patients. Acta Neurologica Scandinavica, 94: 269-275. 